\title{
DYNAMIC EFFECT OF MOVING VEHICLES ON THE ROAD CONCRETE SLABS
}

The road concrete slabs represent the typical transport structure exposed to dynamic effects of moving vehicles. Numerical simulation methods demand to pay attention to the proposal of the computing model of the vehicle and the computing model of the road concrete slab. The models must be mathematically described and the equations of motion must be solved in a numerical way. For the solution of such a problem the specialized computer program must be created. It is suitable to use the possibilities of the higher level programming languages as MATLAB. The influence of some important factors on dynamic response of the structure is numerically tested.

\section{Introduction}

The road structure is the typical transport structure exposed to dynamic effects of moving vehicles. The classic road structures are designed as bitumen pavements. At the present time also the concrete pavements are sometimes utilized in the form of concrete slabs. From the practical point of view it is needed to known the dynamic response of the slab structure on the effect of moving vehicles [1], [2], [3], [4]. One possibility of dynamic analysis is to utilize the methods of numerical simulation. This approach demands to pay attention to the proposal of the computing models of vehicle and the computing models of the road concrete slab. The dynamic response of the road concrete slab is dependent on the parameters of vehicle, the parameters of the slab and the parameters of subgrade. The submitted paper presents the influence of some parameters of the truck and the slab on the dynamic response of analysed structure.

\section{Computing model of vehicle}

Generally the one two or three dimensional computing model of the vehicle can be adopted for the modelling of dynamic effect of moving vehicles on the road structures. For the purpose of the task presented in this paper the plane computing model of the truck TATRA was adopted, Fig. 1.

The computing model of vehicle has 8 degrees of freedom 5 mass and 3 massless. The massless degrees of freedom correspond to the vertical movements of the contact points of the model with the surface of the runway. The vibration of the mass objects of the model is described by the 5 functions of the time $r_{i}(t),(i=1,2$, $3,4,5)$. The massless degrees of freedom are coupled by contact

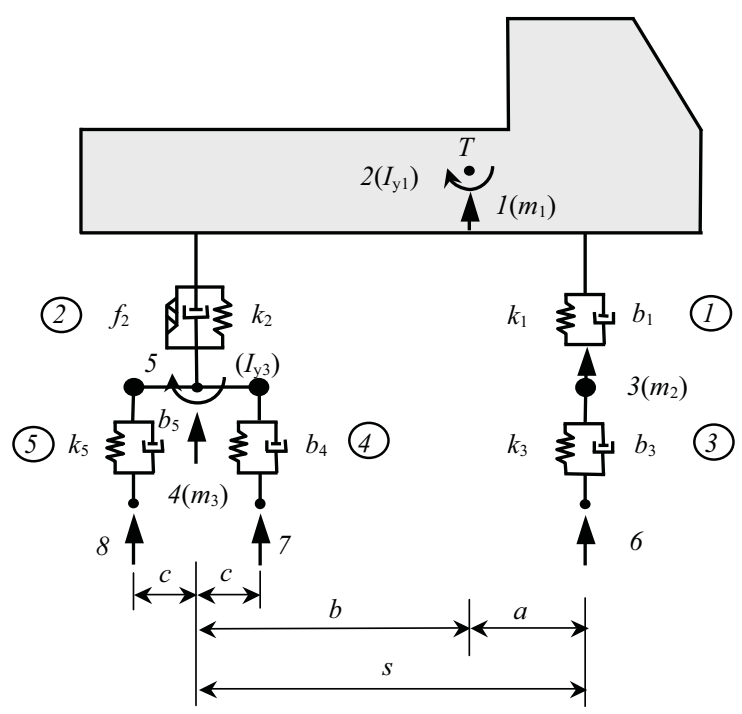

Fig. 1 Plane computing model of the truck TATRA

forces $F_{R V, i}(t),(i=6,7,8$,$) acting at the contact points. The equa-$ tions of motions and the expressions for contact forcers have the following form:

The meaning of the used symbols is as follows (Fig. 1): $k_{i}, b_{i}, f_{i}$ are the stiffness, damping and friction characteristics of the model, $m_{i}, I_{y i}$ are the mass and inertia characteristics of the model, $a, b, c$, $s$ are the length characteristic of the model, $g=9.81 \mathrm{~m} . \mathrm{s}^{-2}, G_{i}$ are the gravity forces acting at the contact points with the surface of the runway. The deformations of the spring elements are $d_{i}(t)$ and the derivation with respect of time is denoted by the dot over the symbol.

\footnotetext{
* Gabriela Lajcakova, Jozef Melcer

Department of Structural Mechanics, Faculty of Civil Engineering, University of Zilina, E-mail: gabriela.lajcakova@fstav.uniza.sk
} 


$$
\begin{aligned}
& \ddot{r}_{1}(t)=-\left\{+k_{1} \cdot d_{1}(t)+b_{1} \cdot \dot{d}_{1}(t)+k_{2} \cdot d_{2}(t)+b_{2} \cdot \dot{d}_{2}(t)+f_{2} \cdot \dot{d}_{2}(t) / \dot{d}_{c}\right\} / m_{1}, \\
& \ddot{r}_{2}(t)=-\left\{-a \cdot k_{1} \cdot d_{1}(t)-a \cdot b_{1} \cdot \dot{d}_{1}(t)+b \cdot k_{2} \cdot d_{2}(t)+b \cdot b_{2} \cdot \dot{d}_{2}(t)+f_{2} \cdot \dot{d}_{2}(t) / \dot{d}_{c}\right\} / I_{y_{1}}, \\
& \left.\ddot{r}_{3}(t)=-\left\{-k_{1} \cdot d_{1}(t)-b_{1} \cdot \dot{d}_{1}(t)+k_{3} \cdot d_{3}(t)+b_{3} \cdot \dot{d}_{3}(t)\right)_{2}\right\} / m_{2}, \\
& \ddot{r}_{4}(t)=-\left\{-k_{2} \cdot d_{2}(t)+b_{2} \cdot \dot{d}_{2}(t)+f_{2} \cdot \dot{d}_{2}(t) / \dot{d}_{c}+k_{4} \cdot d_{4}(t)+b_{4} \cdot \dot{d}_{4}(t)+k_{5} \cdot d_{5}(t)+b_{5} \cdot \dot{d}_{5}(t)\right\} / m_{3}, \\
& \ddot{r}_{5}(t)=-\left\{-c \cdot k_{4} \cdot d_{4}(t)-c \cdot b_{4} \cdot \dot{d}_{4}(t)+c \cdot k_{5} \cdot d_{5}(t)+c \cdot b_{5} \cdot \dot{d}_{5}(t)\right\} / I_{y_{3}}, \\
& F_{6}(t)=G_{6}-k_{3} \cdot d_{3}(t)-b_{3} \cdot \dot{d}_{3}(t)=+g \cdot\left(m_{1} \cdot \frac{b}{s}+m_{2}\right)-k_{3} \cdot d_{3}(t)-b_{3} \cdot \dot{d}_{3}(t), \\
& F_{7}(t)=G_{7}-k_{4} \cdot d_{4}(t)-b_{4} \cdot \dot{d}_{4}(t)=+\frac{1}{2} \cdot g \cdot\left(m_{1} \cdot \frac{a}{s}+m_{3}\right)-k_{4} \cdot d_{4}(t)-b_{4} \cdot \dot{d}_{4}(t), \\
& F_{8}(t)=G_{8}-k_{5} \cdot d_{5}(t)-b_{5} \cdot \dot{d}_{4}(t)=+\frac{1}{2} \cdot g \cdot\left(m_{1} \cdot \frac{a}{s}+m_{3}\right)-k_{5} \cdot d_{5}(t)-b_{5} \cdot \dot{d}_{5}(t) .
\end{aligned}
$$

\section{Computing model of the road slab}

The space computing model of the road concrete slab is based on the Kirchhoff theory of the thin slab resting on Winkler elastic foundation. The equation of the slab is

$$
\begin{aligned}
& D\left(\frac{\partial^{4} w}{\partial x^{4}}+2 \frac{\partial^{4} w}{\partial x^{2} \partial y^{2}}+\frac{\partial^{4} w}{\partial y^{4}}\right)+K \cdot w+\mu \frac{\partial^{2} w}{\partial t^{2}}+ \\
& +2 \mu \omega_{b} \frac{\partial w}{\partial t}=p(x, y, t) .
\end{aligned}
$$

It is the partial differential equation for the unknown function $w(x, y, t)$ representing the bending surface of the slab centre plane. The solution of the differential equation will be realised in the sense of Fourier method. The wanted function $w(x, y, t)$ will be expressed as the product of two functions

$$
w(x, y, t)=w_{o}(x, y) \cdot q(t) .
$$

The function $w_{o}(x, y)$ figures as known function and it is dependent on the coordinates $x, y$ only and the function $q(t)$ figures as unknown function and it is dependent on the time $t$. The function $q(t)$ has the meaning of generalized Lagrange coordinate. With the respect to the goal of the solution the assumption about the shape of the function $w_{o}(x, y)$ was adopted

$$
w_{o}(x, y)=\frac{1}{4}\left(1-\cos \frac{2 \pi x}{l_{x}}\right)\left(1-\cos \frac{2 \pi y}{l_{y}}\right) .
$$

The meaning of the other symbols is as follows: $D$ is the slab stiffness [N.m], $K$ is modulus of foundation in $\left[\mathrm{N} . \mathrm{m}^{-3}\right], \mu$ is the mass intensity $\left[\mathrm{kg} . \mathrm{m}^{-2}\right], \omega_{b}$ is damping circular frequency [rad. $\mathrm{s}^{-1}$ ], $p(x, y, t)$ represent the continuous load in $\left[\mathrm{N} \cdot \mathrm{m}^{-2}\right], l_{x}, l_{y}$ are the length and width of the slab in [m]. With the respect to the assumption (5) for $x=l_{x} / 2, y=l_{y} / 2$ the equation (3) can be rewritten to the form

$$
\begin{aligned}
& \ddot{q}(t) \cdot \mu+\dot{q}(t) \cdot 2 \mu \cdot \omega_{b}+q(t) \cdot \\
& \cdot \frac{D}{2}\left[\left(\frac{2 \pi}{l_{x}}\right)^{4}+\left(\frac{2 \pi}{l_{x}}\right)^{2}\left(\frac{2 \pi}{l_{y}}\right)^{2}+\left(\frac{2 \pi}{l_{y}}\right)^{4}+2 \frac{K}{D}\right]= \\
& =p(x, y, t) .
\end{aligned}
$$

In the case of plane computing model of vehicle the $p(x, y, t)$ can be expressed as

$$
\begin{aligned}
& p(x, y, t)=\sum_{j=6,7,8} F_{j}(t) \cdot \frac{1}{l_{x} l_{y}} . \\
& \left(1-\cos \frac{2 \pi x_{j}}{l_{x}}\right)\left(1-\cos \frac{2 \pi y_{o}}{l_{y}}\right) .
\end{aligned}
$$

$F_{j}(t)$ is the contact force under the $j$-th axle of vehicle at the position $x_{j}$ and $y_{o}$.

\section{Numerical analysis}

For the purpose of numerical analysis the following slab construction was considered, Fig. 2:

1. CS - concrete slab,

$h_{1}=240 \mathrm{~mm}, E_{1}=37500 \mathrm{MPa}, v_{1}=0.20$

2. CA II - coating aggregate, quality class II, $h_{2}=40 \mathrm{~mm}, E_{2}=4500 \mathrm{MPa}, v_{2}=0.21$

3. SC I - soil cement, quality class I,

$h_{3}=200 \mathrm{~mm}, E_{3}=2000 \mathrm{MPa}, v_{3}=0.23$

4. PC - protective coat, gravel sand,

$h_{4}=250 \mathrm{~mm}, E_{4}=120 \mathrm{MPa}, v_{4}=0.35$

5. SS - sub-soil,

$h_{5}=\infty \mathrm{mm}, E_{5}=30 \mathrm{MPa}, v_{5}=0.35$

$\mathrm{CS} ; \quad 240 \mathrm{~mm} ; \mathrm{E}=37500 \mathrm{MPa} ; v=0.20$

CA II; $40 \mathrm{~mm} ; \mathrm{E}=4500 \mathrm{MPa} ; v=0.21$
$\mathrm{SC} \mathrm{I} ; 200 \mathrm{~mm} ; \mathrm{E}=2000 \mathrm{MPa} ; v=0.23$
$\mathrm{PC} ; \quad 250 \mathrm{~mm} ; \mathrm{E}=120 \mathrm{MPa} ; v=0.35$
$\mathrm{SS} ; \quad \infty \mathrm{mm} ; \mathrm{E}=30 \mathrm{MPa} ; v=0.35$

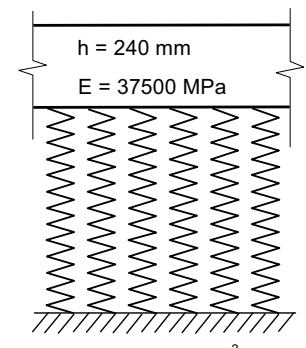

$\mathrm{K}=171,8 \mathrm{MN} / \mathrm{m}^{3}$

Fig. 2 Model of the slab on elastic foundation 
The 1st layer of the computing model is the concrete slab of the thickness $h=240 \mathrm{~mm}$, the length $l_{x}=6.0 \mathrm{~m}$, the width $l_{y}=$ $=3.75 \mathrm{~m}$, modulus of elasticity $E=37500 \mathrm{MPa}$, Poisson coefficient $v=0.20$. The layers under the slab are integrated in the computing model as Winkler foundation. Modulus of foundation $\mathrm{K}$ was calculated by the computer program LAYMED. The parameters of computing model of vehicle correspond to the vehicle TATRA 815 . Total mass of the computing model of vehicle was 13 tons. For the numerical solution of the mathematical apparatus the computer program in program language MATLAB was created. The program enables to calculate the time courses of all kinematic values (deflection, speed, acceleration) at midspan of the slab and the time courses of contact forces under individual axles. The demonstrations of the form of obtained results are in Figs. 3, 4, 5, 6 .

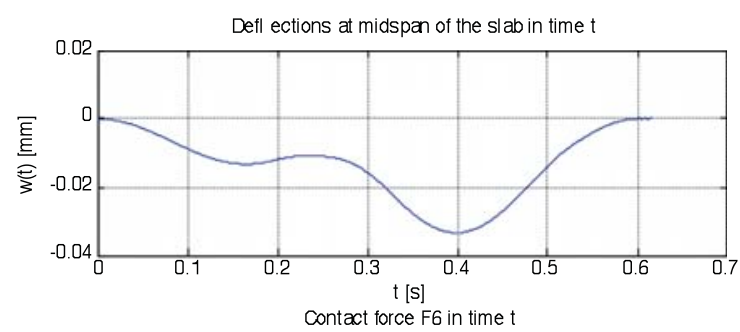

Fig. 3 Time course of the deflection at midspan of the slab

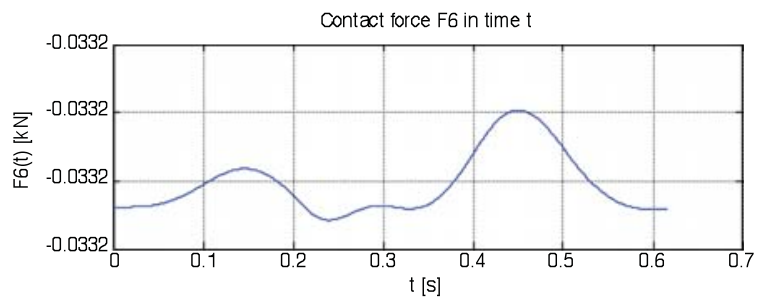

Fig. 4 Time course of the contact force F6 under front axle

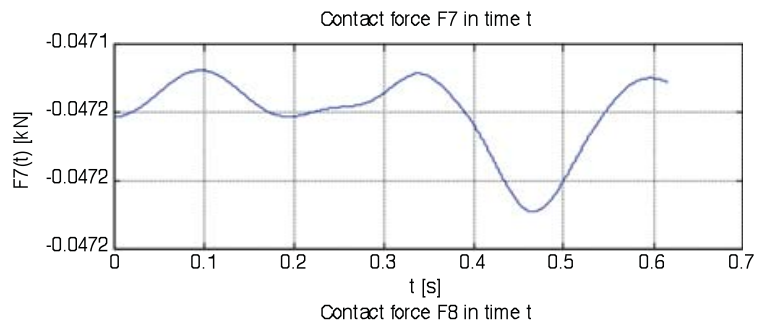

Fig. 5 Time course of the contact force F7 under front wheel of the rear axle

The results of solution are influenced by various parameters of the considered system. In this paper the influence of the speed of vehicle motion and the influence of the modulus of foundation

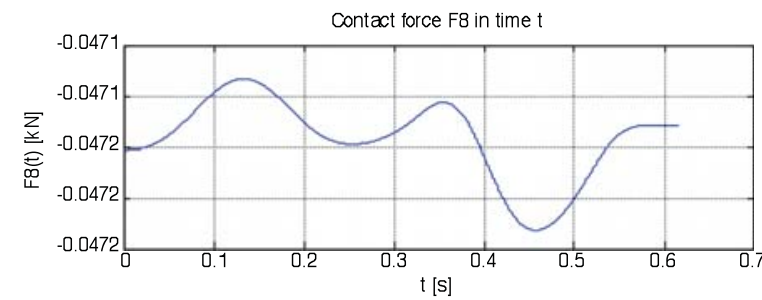

Fig. 6 Time course of the contact force F8 under rear wheel of the rear axle

are analyzed. With the goal to clarify the influence of these two parameters the parametric studies were realized. The influence of the speed of vehicle motion was analyzed in the interval of speeds $V=0-120 \mathrm{~km} / \mathrm{h}$ with the step $5 \mathrm{~km} / \mathrm{h}$. The maximums of vertical deflections at the midspan of the slab versus speed of vehicle motion are plotted in Fig. 7. Similarly the extremes (maximum, minimum) of contact forces under front and rear axles versus speed of vehicle motion are plotted in Figs. 8, 9, 10.

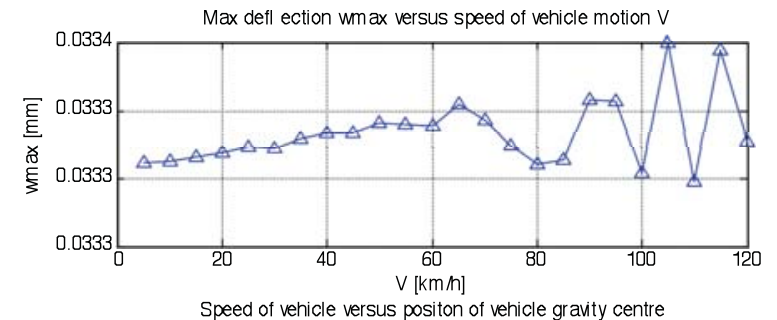

Fig. 7 Maximal deflections at midspan of the slab versus speed of vehicle motion

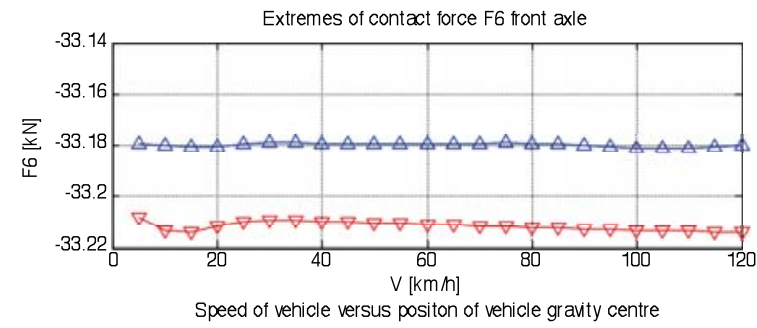

Fig. 8 Extremes of contact force F6 under front axle versus speed of vehicle motion

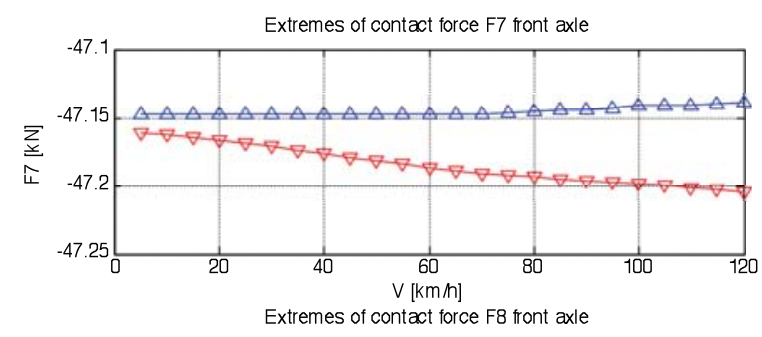

Fig. 9 Extremes of contact force F7 under front wheel of rear axle versus speed of vehicle motion 


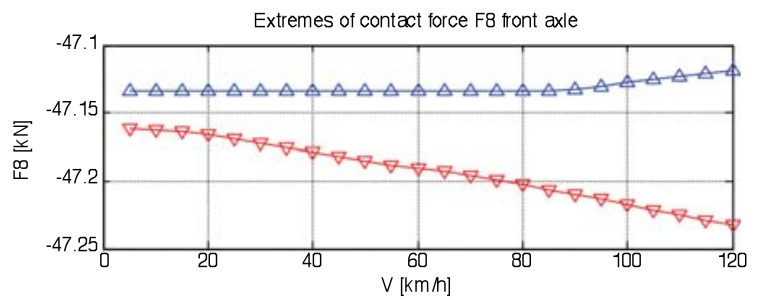

Fig. 10 Extremes of contact force F8 under rear wheel of rear axle versus speed of vehicle motion

The influence of the modulus of foundation $K$ was analyzed in the interval $50.10^{6}-200.10^{6} \mathrm{MN} / \mathrm{m}^{3}$ with the step $25.10^{6} \mathrm{MN} / \mathrm{m}^{3}$ and in the interval $200.10^{6}-500.10^{6} \mathrm{MN} / \mathrm{m}^{3}$ with the step $50.10^{6}$ $\mathrm{MN} / \mathrm{m}^{3}$. The speed of vehicle motion was $V=65 \mathrm{~km} / \mathrm{h}$. The maximums of vertical deflections at the midspan of the slab versus modulus of foundation are plotted in Fig. 11. Similarly the extremes (maximum, minimum) of contact forces under front and rear axles versus modulus of foundation are plotted in Figs. 12, 13, 14.

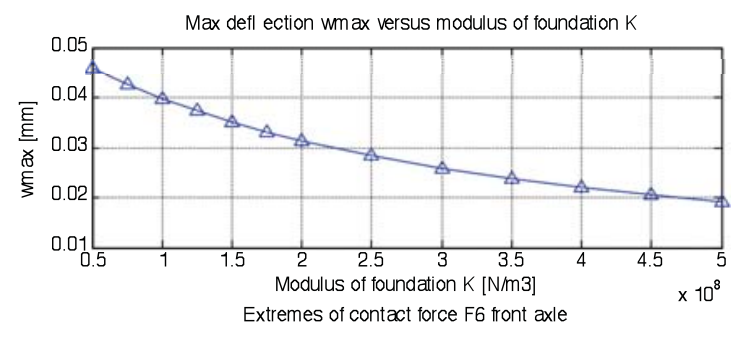

Fig. 11 Maximal deflections at midspan of the slab versus modulus of foundation

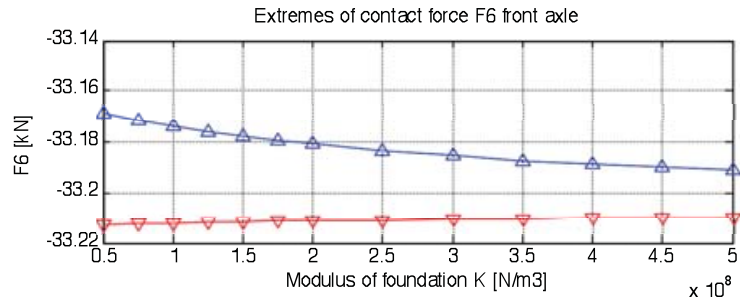

Fig. 12 Extremes of contact force F6 under front axle versus modulus of foundation

\section{Conclusion}

Vehicle-road dynamic interaction is a topical engineering problem. The basic assumption of numerical solution is the creation of computing models. The computing models of vehicles are usually created as discrete computing models described by the system of ordinary differential equations. Minor attention is dedicated to the creation of pavement computing models. One possibility is to model the pavement as the slab on elastic foundation. Such computing

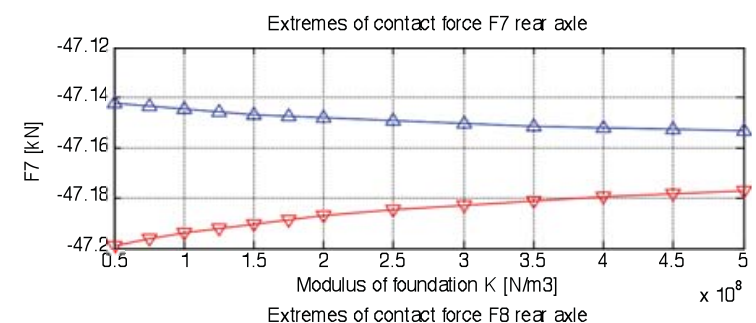

Fig. 13 Extremes of contact force $F 7$ under front wheel of rear axle versus modulus of foundation

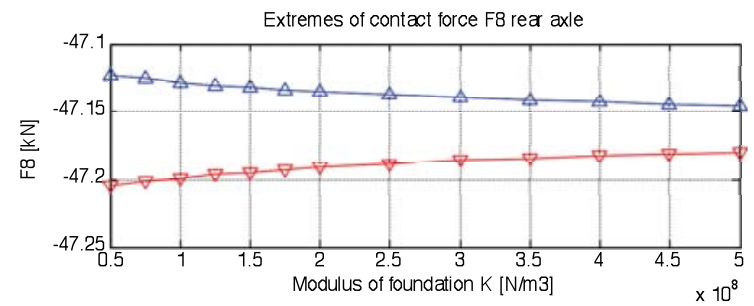

Fig. 14 Extremes of contact force F8 under rear wheel of rear axle versus modulus of foundation

model is suitable for modeling the dynamic effect of moving vehicles on the concrete pavements. When we want to follow the dynamic effect of moving vehicles in one point only we can adopt the assumption about shape of deflection surface of the slab. On the basis of this assumption we can replace the partial differential equation of the slab by ordinary differential equation. Such a computing model enables to analyze all kinematic values (displacement, speed, acceleration) of vehicle and the slab and contact forces acting on the slab. Various parameters come into the analysis. In this paper the influence of speed of vehicle motion and influence of modulus of foundation on the obtained results were analyzed. The deflections of the slab and the extremes of contact forces grow with the speed of vehicle motion. When the slab has good surface without evident unevenness the influence of the speed of vehicle motion on the kinematical and force quantities is very small. The influence of foundation modulus has the opposite tendency as the influence of speed of vehicle motion. The deflections of the slab and the extremes of contact forces fall with the grow values of foundation modulus. The analysis in time domain can be extended on the analysis in frequency domain. The results in frequency domain can serve for the analysis of noise emissions around the roads [5] or for the analysis of seismic problems due to transport [6].

\section{Acknowledgements}

This work was supported by the Slovak Grant National Agency VEGA, grant G1/0031/09.

This contribution is the result of the project implementation: "Centre of Excellence in Transport Engineering" (ITMS: 26220120027) supported by the Research \& Development Operational Programme funded by the ERDF. 


\section{COMMNICOIIONS}

\section{References}

[1] MARTINCEK, G.: Dynamic Diagnostics of Pavements (in Slovak). VEDA, Bratislava, 1983.

[2] MARTINCEK, G.: Dynamics of Pavement Structures, E \& FN Spon and Ister Science Press, London/Bratislava, 1994.

[3] CEBON, D.: Handbook of Vehicle - Road Interaction, Swets\&Zeitlinger Publishers, Lisse, Netherlands, 1999.

[4] KUlaKowSKI, B. T., GARDNER, J. F., LOWEN, S. J.: Dynamic Modeling and Control of Engineering Systems, The $3^{\text {rd }}$ edition, Cambridge University Press, New York, 2009.

[5] PANULINOVA, E.: The Influence of the Road Surface Unevenness on the Noise Level from Automobile Transport (in Slovak). Silnicni obzor, Vol. 62, No. 11,12, 2001, Praha, ISSN 0322-7154, p. 275-279.

[6] KOTRASOVA, K.: Seismic Proposal of Rectangular Tanks Filled by Water (in Slovak). Civil and Environmental Engineering, Vol. 4, No.1, 2008, ISSN 1336-5935, p. 46-53. 